\title{
GROUPS OF HOMOLOGICAL DIMENSION ONE
}

\author{
IOANNIS EMMANOUIL
}

\begin{abstract}
We report on recent work concerning groups of homological dimension one and detail some methods that may be used in order to determine whether these groups are locally free.
\end{abstract}

\section{INTRODUCTION}

Stallings has established in [20] a characterization of finitely generated free groups, as those groups whose cohomological dimension is one. It is very easy to show that a free group has cohomological dimension one. Indeed, if $G$ is a free group then the augmentation ideal $I_{G}$ is a free $\mathbb{Z} G$-module; in fact, if $G$ is freely generated by a subset $S$, then $I_{G}$ is a free $\mathbb{Z} G$-module on the set $\{s-1: s \in S\}$. The essence of Stallings' theorem is that the converse implication is also true, namely that any finitely generated group of cohomological dimension one is free. Bieri asked in [2] whether a (stronger) homological version of the latter result holds:

Is any finitely generated group of homological dimension one free?

Shortly after the publication of the proof of Stallings' theorem, Swan showed that the finite generation hypothesis is redundant therein, by proving that a (not necessarily finitely generated) group $G$ is free if and only if $\mathrm{cd} G=1$ (cf. [21]). In that direction, we note that Bieri's question may be equivalently formulated as follows:

\section{Is any group of homological dimension one locally free?}

Some interesting results concerning that problem have been obtained in [5] and [11], by embedding the integral group ring $\mathbb{Z} G$ of the group $G$ into the associated von Neumann algebra $\mathcal{N} G$ and the algebra $\mathcal{U} G$ of unbounded operators which are affiliated to $\mathcal{N} G$.

We note that a group $G$ is known to be finitely generated if and only if the augmentation ideal $I_{G}$ is a finitely generated $\mathbb{Z} G$-module, whereas $G$ has homological dimension one if and only if $I_{G}$ is a flat $\mathbb{Z} G$-module. In view of the above mentioned result of Stallings and Swan, the freeness of $G$ is equivalent to the projectivity of $I_{G}$ as a $\mathbb{Z} G$-module. Therefore, Bieri's question turns out to be equivalent to the following one:

\section{If the augmentation ideal $I_{G}$ is a finitely generated flat $\mathbb{Z} G$-module,} then is it true that $I_{G}$ is a projective $\mathbb{Z} G$-module?

If $G$ is any countable group, then the augmentation ideal $I_{G}$ is countably presented as a $\mathbb{Z} G$ module. It follows from a result of Lazard [12] that, in this case, the flatness of $I_{G}$ implies that its projective dimension is $\leq 1$ (and hence that $\mathrm{cd} G \leq 2$ ). The point is to show that if we strengthen the assumption on the group $G$ and assume that it is finitely generated (and not just countable), then the flatness of $I_{G}$ implies its projectivity. In this direction, we note that if $G$ is the additive group of rational numbers, then the $\mathbb{Z} G$-module $I_{G}$ is flat but not projective (i.e. hd $G=1$ but $\mathrm{cd} G=2$ ); even though this countable group has homological dimension one, it isn't free.

Research supported by a GSRT/Greece excellence grant, cofunded by the ESF/EU and National Resources. 
In the present note, we shall follow [6] and elaborate on two methods that may be used in order to study this problem.

\section{Projectivity of Finitely Generated flat Modules}

Let $G$ be a group and consider the augmentation ideal $I_{G}$. As mentioned in the Introduction, we wish to prove that the $\mathbb{Z} G$-module $I_{G}$ is projective, provided that we know it to be finitely generated and flat. We are only interested in the very particular $\mathbb{Z} G$-module $I_{G}$, but it may be the case that our group $G$ is such that any finitely generated flat $\mathbb{Z} G$-module is projective. Even though it seems that we make the problem unnecessarily harder ${ }^{1}$, we introduce the following class of groups:

Definition 1.1. Let $\mathfrak{S}$ be the class consisting of those groups $G$, which are such that any finitely generated flat $\mathbb{Z} G$-module is projective.

We shall begin by listing a couple of elementary properties of this class:

(i) $\mathfrak{S}$ contains all finite groups.

Proof. If $G$ is a finite group, then the group $\operatorname{ring} \mathbb{Z} G$ is (left) Noetherian and hence any finitely generated $\mathbb{Z} G$-module is finitely presented. In particular, any finitely generated flat $\mathbb{Z} G$-module is (finitely presented and flat and hence) projective.

(ii) $\mathfrak{S}$ is subgroup-closed.

Proof. Let $H$ be a subgroup of a group $G \in \mathfrak{S}$. In order to show that $H \in \mathfrak{S}$, let $M$ be a finitely generated flat $\mathbb{Z} H$-module. Then, $\operatorname{ind}_{H}^{G} M$ is a finitely generated flat $\mathbb{Z} G$-module and hence, in view of the hypothesis made on $G$, it is $\mathbb{Z} G$-projective. Therefore, the $\mathbb{Z} H$-module $\operatorname{res}_{H}^{G} \operatorname{ind}_{H}^{G} M$ is projective. Since $M$ is a direct summand of $\operatorname{res}_{H}^{G} \operatorname{ind}_{H}^{G} M$, it follows that $M$ is a projective $\mathbb{Z} H$-module as well.

(iii) Any $\mathfrak{S}$-group of homological dimension one is locally free.

Proof. Let $H$ be a finitely generated subgroup of an $\mathfrak{S}$-group $G$. Since $G$ has homological dimension one, it follows that $H$ has homological dimension one as well; hence, the augmentation ideal $I_{H}$ is a finitely generated flat $\mathbb{Z} H$-module. Since the class $\mathfrak{S}$ is subgroup-closed (cf. (ii) above), the group $H$ is contained in $\mathfrak{S}$ and hence the $\mathbb{Z} H$-module $I_{H}$ is projective. Invoking Stallings' theorem, we conclude that $H$ is a free group, as needed.

Remark 1.2. The class of those rings $R$ over which any finitely generated flat left module is projective has been studied in [8] and [18]. It is shown therein that this property is equivalent to the ascending chain condition on certain sequences of principal left ideals in matrix rings over $R$. In particular, it turns out that a group $G$ is an $\mathfrak{S}$-group if and only if for any $n \geq 1$ and any sequence of $n \times n$ matrices $\left(A_{i}\right)_{i} \in \mathbf{M}_{n}(\mathbb{Z} G)$ with $A_{i} A_{i+1}=A_{i}$ for all $i$, the ascending sequence of principal left ideals

$$
\mathbf{M}_{n}(\mathbb{Z} G) A_{0} \subseteq \mathbf{M}_{n}(\mathbb{Z} G) A_{1} \subseteq \ldots \subseteq \mathbf{M}_{n}(\mathbb{Z} G) A_{t} \subseteq \cdots
$$

of the matrix ring $\mathbf{M}_{n}(\mathbb{Z} G)$ is eventually constant.

The embedding of the integral group ring $\mathbb{Z} G$ of a group $G$ into the associated von Neumann

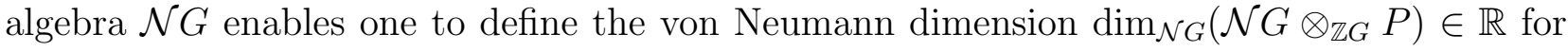

\footnotetext{
${ }^{1}$ See Remark 1.8(ii) below.
} 
any finitely generated projective $\mathbb{Z} G$-module $P$. The reader may consult Lück's book [14] for the details of the definition and several useful properties of this dimension theory.

For any group $G$ we let $\Lambda_{G}$ be the additive subgroup of $\mathbb{Q}$, which is generated by the inverses of the orders of the finite subgroups of $G$. The group $G$ is said to satisfy Atiyah's conjecture if $\operatorname{dim}_{\mathcal{N} G}\left(\mathcal{N} G \otimes_{\mathbb{Z} G} P\right) \in \Lambda_{G}$ for any finitely generated projective $\mathbb{Z} G$-module $P$. Lück's book contains a thorough discussion of this conjecture and presents several classes of groups for which the conjecture is known to be true.

Definition 1.3. Let $\mathfrak{A}_{\text {fin }}$ be the class consisting of those groups that satisfy Atiyah's conjecture and have a finite upper bound on the orders of their finite subgroups.

The relevance of Atiyah's conjecture in the study of groups of homological dimension one was noticed by Kropholler, Linnell and Lück in [11]: In order to describe the link between the two themes, let us consider a finitely generated group $G$ and fix a presentation of it as the quotient of a finitely generated free group $\Gamma$ by a normal subgroup $N$. Then, as shown by Magnus in [16], the associated relation module $P=N /[N, N]$ fits into a short exact sequence of $\mathbb{Z} G$-modules

$$
0 \longrightarrow P \longrightarrow F \longrightarrow I_{G} \longrightarrow 0,
$$

where $F$ is a finitely generated free $\mathbb{Z} G$-module (of rank equal to the rank of the free group $\Gamma$ ); see also [3, Chapter II, Proposition 5.4]. If the group $G$ has homological dimension one, then the augmentation ideal $I_{G}$ is flat as a $\mathbb{Z} G$-module. Invoking [12, Théorème I.3.2], we conclude that $I_{G}$ has projective dimension $\leq 1$ and hence $P$ is a projective $\mathbb{Z} G$-module. Then, since the (finitely generated flat) $\mathbb{Z} G$-module $I_{G}$ is projective if and only if it is finitely presented, in order to prove the conjectured freeness of the group $G$, we are reduced to showing that the relation module $P$ is finitely generated.

It is well known that if we are dealing with vector spaces over a field, then any subspace of a finitely generated vector space $V$ is also finitely generated, i.e. there is no strictly increasing sequence of subspaces of $V$. One may prove this property using the fact that the dimension of any such a subspace is bounded by the dimension of $V$ and hence the dimensions of these subspaces may assume only finitely many values. In the same way, one may attempt to prove that the $\mathbb{Z} G$-module $P$ in the exact sequence (1) above is finitely generated, using the formal properties of the von Neumann dimension (namely, its monotonicity and continuity). There is a problem though with this approach: The von Neumann dimension is a real number (not necessarily an integer) and a bounded set of real numbers need not be discrete. Atiyah's conjecture for groups with a finite upper bound on the orders of their finite subgroups guarantees that the set of the von Neumann dimensions that are involved is indeed discrete, making the classical linear algebra argument outlined above work.

In fact, we may state the following result, which is essentially due to Kropholler, Linnell and Lück (cf. [11]).

Theorem 1.4. The class $\mathfrak{A}_{\text {fin }}$ is a subclass of $\mathfrak{S}$.

Proof. Let $G$ be a group contained in $\mathfrak{A}_{\text {fin }}$. In order to show that $G$ is an $\mathfrak{S}$-group, it suffices, in view of [8, Proposition 3.5], to show that any finitely generated and countably presented flat $\mathbb{Z} G$-module is projective. To that end, let $M$ be a finitely generated and countably presented flat $\mathbb{Z} G$-module. Being countably presented and flat, the module $M$ has projective dimension $\leq 1$ (cf. [12, Théorème I.3.2]) and hence the arguments used in the proof of [11, Lemma 4 , Lemma 5 and Theorem 2] show that $M$ is finitely presented and therefore projective. 
For any ideal $I$ of a ring $R$ we define the ideal $I^{\omega}=\bigcap_{n=1}^{\infty} I^{n}$. We also consider the ideals $I^{\omega^{m}}$, $m \geq 1$, which are defined inductively by letting $I^{\omega^{1}}=I^{\omega}$ and $I^{\omega^{m}}=\left(I^{\omega^{m-1}}\right) \omega$ for all $m>1$. We say that the ideal $I$ is residually nilpotent if $I^{\omega}=0$.

Definition 1.5. For all $m \geq 1$ let $\mathfrak{X}_{m}$ be the class consisting of those groups $G$ whose augmentation ideal $I_{G}$ is such that $I_{G}^{\omega^{m}}=0$. We also let $\mathfrak{X}=\bigcup_{m=1}^{\infty} \mathfrak{X}_{m}$.

The class $\mathfrak{X}_{1}$, which contains those groups whose augmentation ideal is residually nilpotent, has been studied extensively (cf. [17]). As shown by Lichtman in [13], $\mathfrak{X}_{1}$ contains as a subclass the class

$$
\mathfrak{Y}_{1}=\{\text { residually torsion-free nilpotent groups }\} \text {. }
$$

In particular, $\mathfrak{X}_{1}$ contains all free groups (cf. [15, §I.10]) and all torsion-free abelian groups. As shown in [6, Proposition 5.2], the class $\mathfrak{X}$ is closed under subgroups and extensions. ${ }^{2}$ It follows that $\mathfrak{X}$ contains as a subclass the class

$$
\mathfrak{Y}=\{\text { iterated extensions of residually torsion-free nilpotent groups }\} \text {. }
$$

The relevance of class $\mathfrak{X}$ in the study of $\mathfrak{S}$-groups (and therefore in the study of groups of homological dimension one) stems from the following lifting result, which is itself proved in [6, Proposition 5.3(ii)].

Theorem 1.6. Let $G$ be a group and $N \subseteq G$ a normal subgroup. If $N \in \mathfrak{X}$ and $G / N \in \mathfrak{S}$, then $G \in \mathfrak{S}$.

In particular, the trivial group being an $\mathfrak{S}$-group, it follows that $\mathfrak{X} \subseteq \mathfrak{S}$.

Corollary 1.7. Let $G$ be a group having a normal subgroup $N \subseteq G$, such that:

(i) $N$ is an iterated extension of residually torsion-free nilpotent groups and

(ii) G/N satisfies Atiyah's conjecture and has a finite upper bound on the orders of its finite subgroups.

Then, $G \in \mathfrak{S}$. In particular, if $G$ has homological dimension one, then $G$ is locally free.

Proof. Since $N \in \mathfrak{Y} \subseteq \mathfrak{X}$ and $G / N \in \mathfrak{A}_{\text {fin }} \subseteq \mathfrak{S}$ (cf. Theorem 1.4), the result is an immediate consequence of Theorem 1.6.

Remarks 1.8. (i) (cf. [6, Theorem 5.5]) If $\mathfrak{O}$ is any class of groups, let us denote by $\mathfrak{O}^{\diamond}$ the class consisting of the finitely generated $\mathfrak{O}$-groups of homological dimension one. For example, if we denote by $\mathfrak{F}$ the class of free groups, then $\mathfrak{F}^{\diamond}$ is the class consisting of the free groups of finite rank. With this notation, the chains of inclusions

$$
\mathfrak{F} \subseteq \mathfrak{Y}_{1} \subseteq \mathfrak{X}_{1} \subseteq \mathfrak{X} \subseteq \mathfrak{S} \text { and } \mathfrak{F} \subseteq \mathfrak{Y}_{1} \subseteq \mathfrak{Y} \subseteq \mathfrak{X} \subseteq \mathfrak{S}
$$

induce equalities

$$
\mathfrak{F}^{\diamond}=\mathfrak{Y}_{1}^{\diamond}=\mathfrak{X}_{1}^{\diamond}=\mathfrak{X}^{\diamond}=\mathfrak{S}^{\diamond} \text { and } \mathfrak{F}^{\diamond}=\mathfrak{Y}_{1}^{\diamond}=\mathfrak{Y}^{\diamond}=\mathfrak{X}^{\diamond}=\mathfrak{S}^{\diamond}
$$

Indeed, as we have noted above, a finitely generated $\mathfrak{S}$-group of homological dimension one is necessarily free.

(ii) Let $G$ be a finitely generated group of homological dimension one, so that the augmentation ideal $I_{G}$ is a finitely generated flat $\mathbb{Z} G$-module. If that particular module (namely $I_{G}$ ) is projective, then all finitely generated flat $\mathbb{Z} G$-modules are projective, i.e. $G$ is an $\mathfrak{S}$-group.

\footnotetext{
${ }^{2}$ In fact, the class $\mathfrak{X}_{m}$ is subgroup-closed for all $m$, whereas if $N$ is a normal subgroup of a group $G$, such that $N \in \mathfrak{X}_{m}$ and $G / N \in \mathfrak{X}_{m^{\prime}}$, then $G \in \mathfrak{X}_{m+m^{\prime}}$.
} 
Indeed, the projectivity of $I_{G}$ implies, in view of Stallings' theorem, that the group $G$ is free and hence the inclusion $\mathfrak{F} \subseteq \mathfrak{S}$ (cf. (i) above) shows that $G \in \mathfrak{S}$.

(iii) It is known that $\mathfrak{Y}_{1}$-groups admit a left order. If $G$ is a left orderable group of homological dimension one, then, as shown by Dicks and Linnell in [5, Corollary 6.12], any 2-generated subgroup of $G$ is free. It follows from (i) above that the same result holds for any finitely generated subgroup of a $\mathfrak{Y}_{1}$-group of homological dimension one.

\section{The Cohomology group $H^{2}(G, \mathbb{Z} G)$}

The relation between flatness and projectivity is a classical theme in homological algebra. There is a variety of conditions which, when imposed on a flat module, imply its projectivity. In this direction, it is worth mentioning a result due to Raynaud and Gruson. Even though they were mainly interested in modules over commutative rings (and, more generally, quasicoherent sheaves on schemes), they established in [19] a criterion for a countably presented flat left module $K$ over a not necessarily commutative ring $R$ to be projective. As shown by Lazard $[12, \S I .3]$, such a module $K$ can be expressed as the direct limit of a direct system $\left(K_{n}\right)_{n}$ of finitely generated free left $R$-modules. With this notation, Raynaud and Gruson proved that $K$ is projective if and only if the inverse system of abelian groups $\left(\operatorname{Hom}_{R}\left(K_{n}, R\right)\right)_{n}$ satisfies the Mittag-Leffler condition. The corresponding notion of Mittag-Leffler modules has been studied thoroughly and systematically in [1]. On the other hand, Gray has obtained in [9] a characterization of the Mittag-Leffler condition for inverse systems of countable abelian groups in terms of the vanishing of $\lim ^{1}$, the first derived functor of the inverse limit functor. Using this circle of ideas and the classical expression of the Ext-groups of $K$ in terms of the Extgroups of the $K_{n}$ 's, Jensen has obtained in [10] a simple projectivity test for a flat module, that we shall describe below (see also [6, Theorem 1.3]).

To that end, assume that $R$ is a countable ring and consider a countable left $R$-module $K$. Then, Jensen's criterion asserts that $K$ is projective if and only if $K$ is flat and $\operatorname{Ext}_{R}^{1}(K, R)=0$. A stronger version of that result was obtained in [7, Theorem 2.22], where it is shown that

$$
K \text { is projective } \Longleftrightarrow \operatorname{pd}_{R} K \leq 1 \text { and } \operatorname{Ext}_{R}^{1}(K, R)=0 \text {. }
$$

Let us now consider a non-zero countable left $R$-module $M$ of finite projective dimension. Then, the functors $\operatorname{Ext}_{R}^{i}\left(M,{ }_{-}\right)$vanish identically for $i \gg 0$. In particular, $\operatorname{Ext}_{R}^{i}(M, R)=0$ for $i \gg 0$. Applying the result above to a suitable kernel in a countably generated free resolution of $M$ (namely, to the $(n-1)$ th kernel, where $n=\operatorname{pd}_{R} M$ ), we obtain the equality

$$
\operatorname{pd}_{R} M=\max \left\{i \geq 0: \operatorname{Ext}_{R}^{i}(M, R) \neq 0\right\} \text {. }
$$

The latter equality is of some interest, as it shows that any countable module of finite projective dimension over a countable ring demonstrates a cohomological behavior reminiscent of the $F P$ condition. Indeed, if $R$ is any ring and $M$ any non-zero left $R$-module with $\operatorname{pd}_{R} M=n<\infty$, then the functor $\operatorname{Ext}_{R}^{n}\left(M,{ }_{-}\right)$does not vanish identically, whereas $\operatorname{Ext}_{R}^{i}\left(M,_{-}\right)=0$ for all $i>n$. In fact, if $P_{*} \longrightarrow M \longrightarrow 0$ is a projective resolution of $M$ of length $n$, then it is easily seen that $\operatorname{Ext}_{R}^{n}\left(M, P_{n}\right) \neq 0$. Since $P_{n}$ is a direct summand of a free left $R$-module $F$, it follows that we also have $\operatorname{Ext}_{R}^{n}(M, F) \neq 0$. The free module $F$ is, in general, of infinite rank. In order to obtain some information on the minimal size of such an $F$, we note that:

(i) If $\kappa$ is an infinite cardinal, such that any left ideal of $R$ is generated by a set of cardinality $\kappa$ and $M$ is $\kappa$-generated, then one may choose $P_{n}$ to be $\kappa$-generated. Therefore, it follows that $\operatorname{Ext}_{R}^{n}\left(M, R^{(\kappa)}\right) \neq 0$. 
(ii) In particular, if the ring $R$ is left $\aleph_{0}$-Noetherian (i.e. if any left ideal of $R$ is countably generated) and $M$ is countably generated, then $\operatorname{Ext}_{R}^{n}\left(M, R^{(\mathbb{N})}\right) \neq 0$.

(iii) If $R$ is any ring and $M$ is an FP-module, then $P_{n}$ (and hence the free module $F$ ) may be chosen to be finitely generated; therefore, in this case, we have $\operatorname{Ext}_{R}^{n}(M, R) \neq 0$.

The essence of equality (2) is that, if $R$ is a countable ring and $M$ a countable left $R$-module of projective dimension $n$, then we may choose the free module $F$ for which $\operatorname{Ext}_{R}^{n}(M, F) \neq 0$ to be the left regular module $R$. In other words, the non-triviality of the group $\operatorname{Ext}_{R}^{n}\left(M, R^{(\mathbb{N})}\right)$ (which itself results from the fact that the pair $(R, M)$ satisfies the hypotheses in (ii) above) may be strengthened to the conclusion that $\operatorname{Ext}_{R}^{n}(M, R) \neq 0$ (which is generically a consequence of the finiteness condition in (iii) above).

We now consider the special case where $R=\mathbb{Z} G$ is the integral group ring of a countable group $G$ and $M=\mathbb{Z}$ (with the trivial action of $G$ ). Assuming that $G$ has finite cohomological dimension, we obtain from (2) the equality

$$
\operatorname{cd} G=\max \left\{i \geq 0: H^{i}(G, \mathbb{Z} G) \neq 0\right\} .
$$

If the countable group $G$ has homological dimension one, then we have cd $G \leq 2$ and hence it follows that, in this case,

$$
\operatorname{cd} G=1 \Longleftrightarrow H^{2}(G, \mathbb{Z} G)=0 .
$$

The latter equivalence may be strengthened, as stated in the next result, which is proved in [6, Theorem 3.1].

Theorem 2.1. Let $G$ be a countable group of homological dimension one. Then, the following conditions are equivalent:

(i) $c d G=1$ (i.e. $G$ is free),

(ii) $H^{2}(G, \mathbb{Z} G)=0$,

(iii) $H^{2}(G, \mathbb{Z} G)$ is a countable group.

Corollary 2.2. (cf. [6, Proposition 3.2]) Let $G$ be a finitely generated group of homological dimension one, which contains a non-trivial finitely generated free group as a normal subgroup. Then, $G$ is a free group.

Proof (sketch). If $1 \neq N \subseteq G$ is a normal subgroup, which is finitely generated and free, then one may use the associated Lyndon-Hochschild-Serre spectral sequence in order to obtain an isomorphism of abelian groups $H^{2}(G, \mathbb{Z} G) \simeq H^{1}\left(G / N, H^{1}(N, \mathbb{Z} G)\right)$. Since both groups $N$ and $G / N$ are finitely generated and $\mathbb{Z} G$ is countable, the abelian group $H^{1}\left(G / N, H^{1}(N, \mathbb{Z} G)\right)$ turns out to be countable as well. The freeness of $G$ then follows invoking Theorem 2.1.

As an application of the result above, we can show that any group of homological dimension one must necessarily share certain properties with locally free groups. In order to state explicitly these properties, let us consider a group $G$ of homological dimension one. Then, as shown in [6, §3], the following hold:

(i) For any non-trivial finitely generated and free subgroup $H \subseteq G$ the normalizer $N_{G}(H)$ of $H$ is locally free and the quotient group $N_{G}(H) / H$ is locally finite.

(ii) If $H \subseteq G$ is an infinite cyclic subgroup, then the normalizer $N_{G}(H)$ coincides with the centralizer $C_{G}(H)$ and is locally infinite cyclic.

(iii) If $G$ is non-abelian, then the center of $G$ is trivial. ${ }^{3}$

\footnotetext{
${ }^{3}$ This result may be alternatively proved using the structure of groups of cohomological dimension $\leq 2$; for such a proof, the reader may consult Cornick's unpublished notes [4].
} 


\section{REFERENCES}

[1] Angeleri-Hügel, L., Herbera, D.: Mittag-Leffler conditions on modules. Ind. Univ. Math. J. 57, 2459-2517 (2008)

[2] Bieri, R.: Homological dimension of discrete groups. Queen Mary College Mathematics Notes. Queen Mary College, London 1976

[3] Brown, K.S.: Cohomology of Groups. (Grad. Texts Math. 87) Berlin Heidelberg New York: Springer 1982

[4] Cornick, J.: On groups of homological dimension one. (unpublished manuscript)

[5] Dicks, W., Linnell, P.: $L^{2}$-Betti numbers of one-relator groups. Math. Ann. 337, 855-874 (2007)

[6] Emmanouil, I., Talelli, O.: On the equality between homological and cohomological dimension of groups. J. reine angew. Math. 664, 55-70 (2012)

[7] Emmanouil, I., Talelli, O.: On the flat length of injective modules. J. London Math. Soc. 84, 408-432 (2011)

[8] Facchini, A., Herbera, D., Sakhajev, I.: Finitely generated flat modules and a characterization of semiperfect rings. Comm. Algebra 31, 4195-4214 (2003)

[9] Gray, B.: Spaces of the same $n$-type, for all $n$. Topology 5, 241-243 (1966)

[10] Jensen, C.U.: Variations on Whitehead's problem and the structure of Ext. Models, Modules and Abelian Groups, 407-414. (Edited by Gobel, R. and Goldsmith, B.) Walter de Gruyter: Berlin, New York 2008

[11] Kropholler, P., Linnell, P., Lück, W.: Groups of small homological dimension and the Atiyah conjecture. Geometry and Cohomology in Group Theory, Durham, July 2003, editors: Bridson, M., Kropholler, P.H. and Leary, I.J., LMS Lecture Notes Series 358, 271-277, Cambridge University Press 2009

[12] Lazard, D.: Autour de la platitude. Bull. Soc. Math. France 97, 81-128 (1969)

[13] Lichtman, A.I.: The residual nilpotency of the augmentation ideal and the residual nilpotency of some classes of groups. Israel J. Math. 26, 276-293 (1977)

[14] Lück, W.: $L^{2}$-invariants: theory and applications to geometry and K-theory. Springer 2002

[15] Lyndon, R.C., Schupp, P.E.: Combinatorial group theory. Ergebnisse der Mathematik und ihrer Grenzgebiete 89, Springer: Berlin, New York 1977

[16] Magnus, W.: On a theorem of Marshall Hall. Ann. Math. 40, 764-768 (1939)

[17] Passi, I.B.S.: Group rings and their augmentation ideals. Lecture Notes in Mathematics 715, Springer: Berlin 1979

[18] Puninski, G., Rothmaler, P.: When every finitely generated flat module is projective. J. Algebra 277, $542-558$ (2004)

[19] Raynaud, M., Gruson, L.: Critères de platitude et de projectivité. Invent. Math. 13, 1-89 (1971)

[20] Stallings, J.R.: On torsion free groups with infinitely many ends. Ann. Math. 88, 312-334 (1968)

[21] Swan, R.G.: Groups of cohomological dimension one. J. Algebra 12, 585-610 (1969)

Department of Mathematics, University of Athens, Athens 15784, Greece

E-mail address: emmanoui@math.uoa.gr 